MATHEMATICS OF COMPUTATION

Volume 67, Number 222, April 1998, Pages 751-758

S 0025-5718(98)00919-3

\title{
THE NUMBER OF LATTICE POINTS IN ALCOVES AND THE EXPONENTS OF THE FINITE WEYL GROUPS
}

\author{
RUEDI SUTER
}

\begin{abstract}
We count lattice points in certain rational simplices associated with an irreducible finite Weyl group $W$ and observe that these numbers are linked to the exponents of $W$.
\end{abstract}

\section{INTRODUCTION}

The main point of this note is to present an (as far as I know) new formula for the exponents of an irreducible finite Weyl group. For basic terminology the reader is referred to any one of the books $[1,3,5]$. In any case, let me recall a few ways of how the exponents occur. Thus let $W$ be an irreducible finite Coxeter group with set of Coxeter generators $S$ and $\operatorname{rank} l=|S|$.

The Coxeter transformation $c:=\prod_{s \in S} s \in W$ is well-defined up to conjugacy, and its order $h=|c|$ is the Coxeter number of $W$. If $\exp 2 \pi i m_{1} / h, \ldots, \exp 2 \pi i m_{l} / h$ with $0<m_{1} \leqslant \cdots \leqslant m_{l}<h$ are the eigenvalues of $c$ in the reflection representation, then the integers $m_{1}, \ldots, m_{l}$ are called the exponents of $W$.

Here is another approach to describe the exponents. Let $\ell: W \rightarrow \mathbb{Z}_{\geqslant 0}$ be the length function. Then

$$
\sum_{w \in W} t^{\ell(w)}=\prod_{i=1}^{l} \frac{1-t^{m_{i}+1}}{1-t}
$$

A third characterization of the exponents comes from the fact that the numbers $m_{1}+1, \ldots, m_{l}+1$ are the degrees of the fundamental polynomial invariants of $W$.

Fourth, the exponents of $W$ can be read off the Poincaré polynomial of the hyperplane arrangement associated with $W$.

Suppose from now on that $W$ is crystallographic. It is thus the Weyl group of a compact, connected Lie group $G$. The manifold $G$ has the same cohomology as a product of odd-dimensional spheres,

$$
H^{*}(G, \mathbb{R}) \cong H^{*}\left(\prod_{i=1}^{l} S^{2 m_{i}+1}, \mathbb{R}\right),
$$

which again determines the exponents.

Still another possibility to describe the exponents in the crystallographic case is as follows. Consider a root system with Weyl group $W$, and let $k_{i}$ be the number of

Received by the editor September 3, 1996.

1991 Mathematics Subject Classification. Primary 20F55; Secondary 05A15, 11P21, 11P83, 17B20, 17B67, 52B20.

Supported by the Swiss National Science Foundation.

(C) 1998 American Mathematical Society 
positive roots of height $i$. (The height of a root is the sum of its coefficients when expressed as a linear combination of simple roots.) Then $\left(m_{l}, \ldots, m_{1}\right)$ is the dual partition to the partition $\left(k_{1}, \ldots, k_{h-1}\right)$.

\section{ANOTher FORMUla FOR THE EXPONENTS}

Now we come to another formula for the exponents of an irreducible finite Weyl group $W$, a formula which involves counting lattice points in a rational simplex $\Delta^{(m)}$. As an aside let me mention that in the past few years considerable progress has been made in the theory of toric varieties and hence in the problem of counting lattice points in integral polyhedra (see $[2,4])$. The counting problem we shall deal with is very simple.

Let me explain the definition of $\Delta^{(m)}$, which also provides some motivation. A reader who is not familiar with semisimple Lie theory may hop to the beginning of the next paragraph. Let $\mathfrak{g}$ be a simple complex Lie algebra whose Weyl group is $W$. (It doesn't matter whether we take $\mathfrak{g}=\mathfrak{s o}_{2 l+1}(\mathbb{C})$ or $\mathfrak{g}=\mathfrak{s p}_{2 l}(\mathbb{C})$ if $W$ is hyperoctahedral.) We choose a Cartan subalgebra $\mathfrak{h}$ and a Borel subalgebra $\mathfrak{b}$ with $\mathfrak{h} \subseteq \mathfrak{b} \subseteq \mathfrak{g}$ and thus get an irreducible finite root system $\Phi \subseteq \mathfrak{h}^{*}$ together with a basis of simple roots $\left(\alpha_{i}\right)_{i=1, \ldots, l}$ and its corresponding set of dominant integral weights $P_{+}=\bigoplus_{i=1}^{l} \mathbb{Z}_{\geqslant 0} \varpi_{i}$, where $\left(\varpi_{i}\right)_{i=1, \ldots, l}$ are the fundamental weights, i. e., the basis of $\mathfrak{h}^{*}$ dual to the basis $\left(\alpha_{i}^{\vee}\right)_{i=1, \ldots, l}$ of simple coroots. The intersection $\Phi \cap P_{+}$ consists of the highest root $\theta$ together with the highest short root $\theta_{\mathrm{s}}$. Of course, $\theta=\theta_{\mathrm{s}}$ if $\mathfrak{g}$ is simply laced. We want to count the elements of

$$
\Delta^{(m)}:=\left\{\lambda \in P_{+} \mid\left\langle\lambda, \theta_{\mathrm{s}}^{\vee}\right\rangle \leqslant m\right\} \quad(m \in \mathbb{Z}) .
$$

The coroot $\theta_{\mathrm{s}}^{\vee}$ is the highest root of the dual root system, and its coefficients with respect to the basis $\left(\alpha_{i}^{\vee}\right)_{i=1, \ldots, l}$, i. e., the numbers $n_{i}:=\left\langle\varpi_{i}, \theta_{\mathrm{s}}^{\vee}\right\rangle(i=1, \ldots, l)$ are sometimes called the marks (of the dual root system). Equivalently, $\left|\Delta^{(m)}\right|$ is the number of dominant integral weights (of fixed null depth) at level $m$ of the (twisted if $\mathfrak{g}$ is non-simply-laced) affine Kac-Moody algebra corresponding to $\mathfrak{g}$. (For the untwisted version one has to replace $\theta_{\mathrm{s}}$ by $\theta$ in the definition of $\Delta^{(m)}$. So instead of having $n_{1}, \ldots, n_{l}$ being the marks, one would take them to be the comarks.)

The upshot of the previous paragraph is that we have $l$ positive integers $n_{1}, \ldots, n_{l}$ associated to $W$. The cardinality $\left|\Delta^{(m)}\right|$ we are interested in is nothing else than the dimension of the $K$-algebra ( $K$ is any field)

$$
K\left[x_{1}, \ldots, x_{l}\right] /(\text { monomials of degree strictly bigger than } m)
$$

with $\operatorname{deg} x_{i}=n_{i}(i=1, \ldots, l)$.

The generating function

$$
\frac{1}{\left(1-t^{n_{1}}\right) \cdots\left(1-t^{n_{l}}\right)}=\sum_{m=0}^{\infty}\left|\Delta^{(m)}-\Delta^{(m-1)}\right| \cdot t^{m}
$$

counts nonnegative integral solutions to the equation $a_{1} n_{1}+\cdots+a_{l} n_{l}=m$. The coefficients we are interested in are the sums of the coefficients of this generating function. Putting $n_{0}:=1$, we have

$$
\frac{1}{\left(1-t^{n_{0}}\right) \cdots\left(1-t^{n_{l}}\right)}=\sum_{m=0}^{\infty}\left|\Delta^{(m)}\right| \cdot t^{m}
$$

We say $n_{0}, \ldots, n_{l}$ are the affine marks of $W$. 
Let $\widetilde{n}:=\operatorname{lcm}\left\{n_{0}, \ldots, n_{l}\right\}$. It is clear that there are polynomials $p_{1}(x), \ldots, p_{\widetilde{n}}(x)$ such that $\left|\Delta^{(m)}\right|=p_{a}(m)$ if $m \equiv a(\bmod \widetilde{n})$ and $m \geqslant 0$. In fact,

$$
\begin{aligned}
\frac{1}{\left(1-t^{n_{0}}\right) \cdots\left(1-t^{n_{l}}\right)} & =\left(\prod_{i=0}^{l} \frac{1-t^{\tilde{n}}}{1-t^{n_{i}}}\right) \frac{1}{\left(1-t^{\tilde{n}}\right)^{l+1}} \\
& =\left(\prod_{i=0}^{l} \sum_{k=0}^{\frac{\tilde{n}}{n_{i}}-1} t^{n_{i} k}\right) \sum_{\mu=0}^{\infty}\left(\begin{array}{c}
\mu+l \\
l
\end{array}\right) t^{\tilde{n} \mu} .
\end{aligned}
$$

Substituting equation (3) into the left hand side of equation (2), we thus have an explicit formula for the numbers $\left|\Delta^{(m)}\right|$ and hence for the polynomials $p_{a}(x)$. (It turns out that $p_{a}(x)=p_{b}(x)$ if $\operatorname{gcd}\{a, \widetilde{n}\}=\operatorname{gcd}\{b, \widetilde{n}\}$. This is not so in general but holds for the special choices for $n_{0}, \ldots, n_{l}$ we have to consider.)

Recall that the order of $W$ can be expressed as $|W|=f \cdot l ! \cdot \prod_{i=1}^{l} n_{i}$, where $f$ is the index of connection, i. e., the determinant of the matrix of Cartan integers. It turns out that $f$ is the number of affine marks of $W$ equal to 1 , i. e., $f=\left|\Delta^{(1)}\right|$ by (2). Indeed, this description of $f$ is a consequence of the formula $|W|=\prod_{i=1}^{l}\left(m_{i}+1\right)$, which follows from equation (1), and the formula (4) with $m=1$ below.

Observation. Let $W$ be an irreducible finite Weyl group with affine marks $n_{0}, \ldots, n_{l}$ and exponents $m_{1}, \ldots, m_{l}$. Define $\left|\Delta^{(m)}\right|$ as in equation (2). Let $f$ be the index of connection of $W$ and $\widetilde{n}:=\operatorname{lcm}\left\{n_{0}, \ldots, n_{l}\right\}$. Then

$$
\left|\Delta^{(m)}\right|=\frac{f}{|W|} \prod_{i=1}^{l}\left(m+m_{i}\right)
$$

for every positive integer $m$ satisfying $\operatorname{gcd}\{m, \widetilde{n}\}=1$.

We are now in the usual situation that an empirically observed result awaits for a conceptual proof.

\section{The tables}

The head of each table shows the affine Coxeter-Dynkin diagram. Its vertices are decorated with the marks $n_{0}, \ldots, n_{l}$. The left column contains the polynomials

\begin{tabular}{|c|c|}
\hline$\widehat{A}_{l}$ & $\widetilde{n}=1$ \\
\hline$\prod_{i=1}^{l}(m+i)$ & 1 \\
\hline
\end{tabular}
$\frac{|W|}{f} \cdot\left|\Delta^{(m)}\right|$, and the number in the right column is $\operatorname{gcd}\{m, \widetilde{n}\}$. The polynomials are ordered by increasing coefficients. 


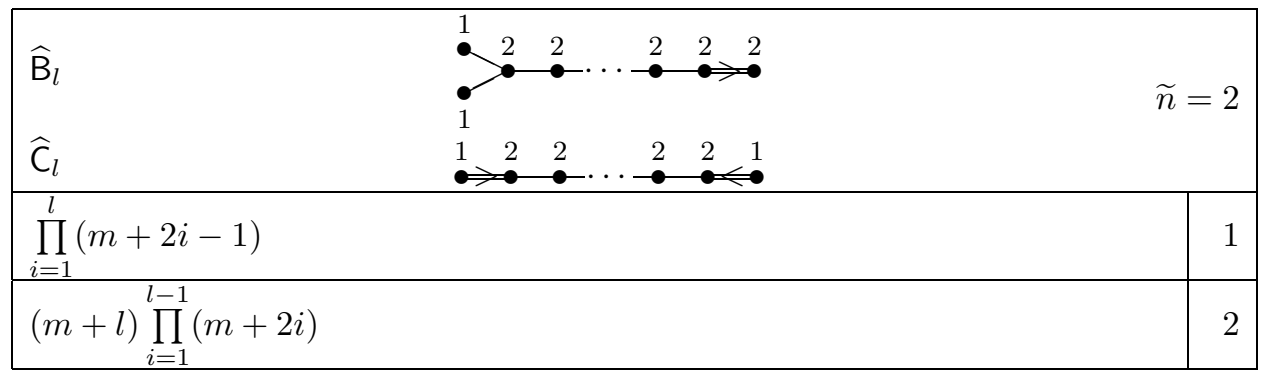

\begin{tabular}{|l|l|}
\hline$\widehat{\mathrm{D}}_{l}$ & \multicolumn{2}{|c|}{$\tilde{n}=2$} \\
\hline$(m+l-1) \prod_{i=1}^{l-1}(m+2 i-1)$ & 1 \\
\hline$\left(m^{2}+2(l-1) m+\frac{l(l-1)}{2}\right) \prod_{i=1}^{l-2}(m+2 i)$ & \\
\hline
\end{tabular}

\begin{tabular}{|l|r|}
\hline & \multicolumn{2}{|c|}{} \\
$\widehat{\mathrm{E}}_{6}$ & $\widetilde{n}=6$ \\
\hline$(m+1)(m+4)(m+5)(m+7)(m+8)(m+11)=$ & 1 \\
$m^{6}+36 m^{5}+510 m^{4}+3600 m^{3}+13089 m^{2}+22284 m+12320$ & \\
\hline$(m+3)(m+9)\left(m^{4}+24 m^{3}+195 m^{2}+612 m+480\right)=$ & \\
$m^{6}+36 m^{5}+510 m^{4}+3600 m^{3}+13089 m^{2}+22284 m+12960$ & 2 \\
\hline$(m+2)(m+4)(m+8)(m+10)\left(m^{2}+12 m+26\right)=$ & \\
$m^{6}+36 m^{5}+510 m^{4}+3600 m^{3}+13224 m^{2}+23904 m+16640$ & \\
\hline$(m+6)^{2}\left(m^{4}+24 m^{3}+186 m^{2}+504 m+480\right)=$ & \\
$m^{6}+36 m^{5}+510 m^{4}+3600 m^{3}+13224 m^{2}+23904 m+17280$ & \\
\hline
\end{tabular}

\begin{tabular}{|l|r|}
\hline$\widehat{\mathrm{E}}_{7}$ & \multicolumn{2}{|c|}{$\tilde{n}=12$} \\
\hline$(m+1)(m+5)(m+7)(m+9)(m+11)(m+13)(m+17)=$ & \\
$m^{7}+63 m^{6}+1617 m^{5}+21735 m^{4}+162939 m^{3}+663957 m^{2}+1286963 m+765765$ & \\
\hline$(m+3)(m+9)(m+15)\left(m^{4}+36 m^{3}+438 m^{2}+2052 m+2289\right)=$ & 3 \\
$m^{7}+63 m^{6}+1617 m^{5}+21735 m^{4}+162939 m^{3}+663957 m^{2}+1304883 m+927045$ & \\
\hline$(m+2)(m+10)(m+13)(m+14)\left(m^{3}+24 m^{2}+155 m+342\right)=$ & 2 \\
$m^{7}+63 m^{6}+1617 m^{5}+21735 m^{4}+163884 m^{3}+689472 m^{2}+1495808 m+1244880$ & \\
\hline$(m+4)(m+5)(m+8)(m+16)\left(m^{3}+30 m^{2}+263 m+504\right)=$ & 4 \\
$m^{7}+63 m^{6}+1617 m^{5}+21735 m^{4}+163884 m^{3}+689472 m^{2}+1495808 m+1290240$ & \\
\hline$(m+6)\left(m^{6}+57 m^{5}+1275 m^{4}+14085 m^{3}+79374 m^{2}+213228 m+234360\right)=$ & 6 \\
$m^{7}+63 m^{6}+1617 m^{5}+21735 m^{4}+163884 m^{3}+689472 m^{2}+1513728 m+1406160$ & \\
\hline$(m+12)\left(m^{6}+51 m^{5}+1005 m^{4}+9675 m^{3}+47784 m^{2}+116064 m+120960\right)=$ & 12 \\
$m^{7}+63 m^{6}+1617 m^{5}+21735 m^{4}+163884 m^{3}+689472 m^{2}+1513728 m+1451520$ & \\
\hline
\end{tabular}




\begin{tabular}{|c|c|c|c|c|c|c|c|c|c|c|c|c|}
\hline & & 10 & $m$ & 10 & $N$ & $\stackrel{?}{-1}$ & 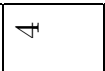 & $\stackrel{\curvearrowright}{\sim}$ & 0 & 穴 & $\mathcal{I}$ & 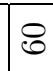 \\
\hline 〈Ш & 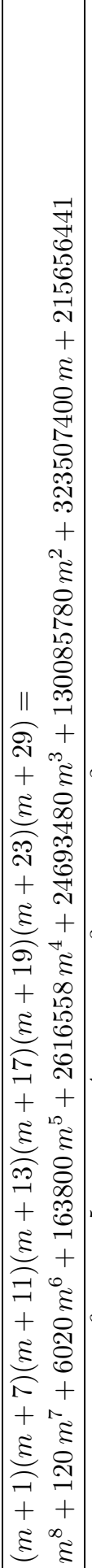 & 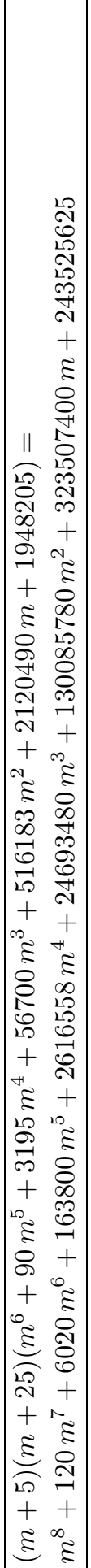 & 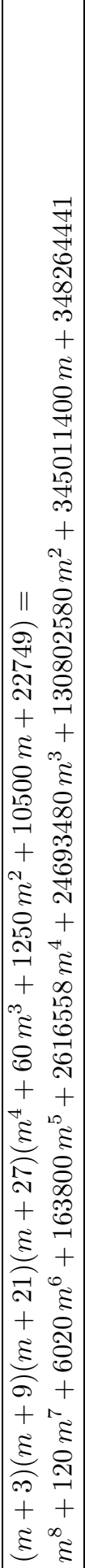 & 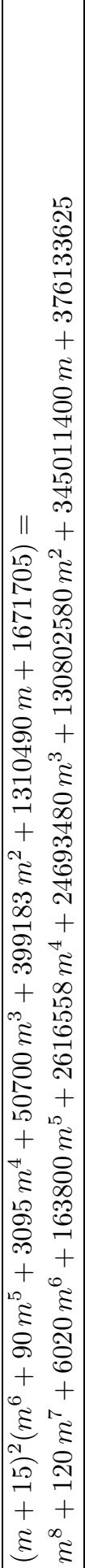 & 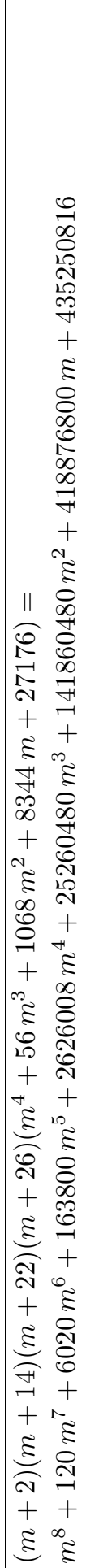 & 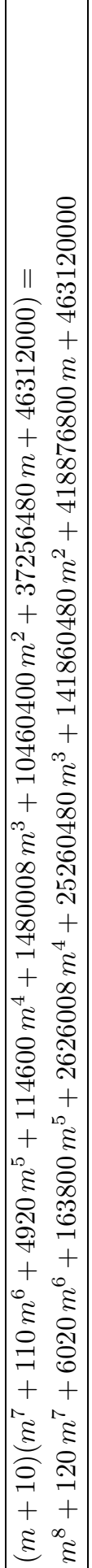 & 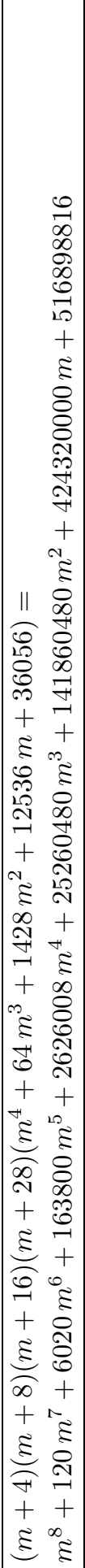 & 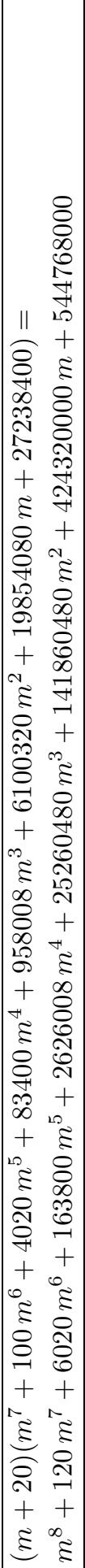 & 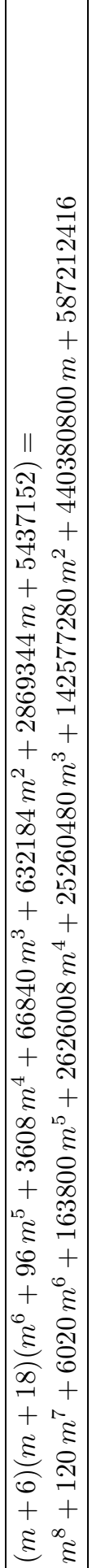 & 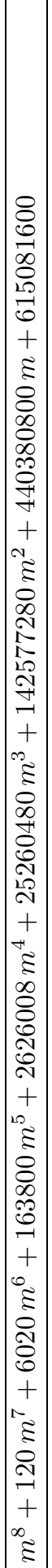 & 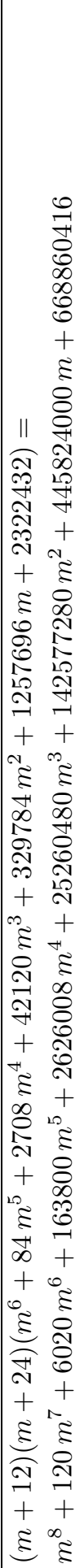 & 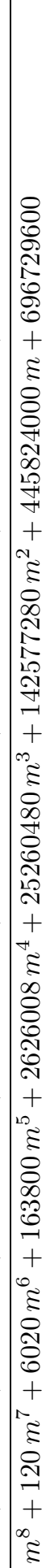 \\
\hline
\end{tabular}




\begin{tabular}{|c|c|c|}
\hline$\widehat{\mathrm{F}}_{4}$ & \begin{tabular}{lll}
3 & 4 & 2 \\
\hdashline & 0
\end{tabular} & $\widetilde{n}=12$ \\
\hline$(m+1)(m+5)(m+7)(m+$ & $m^{4}+24 m^{3}+190 m^{2}+552 m+385$ & \\
\hline$(m+3)(m+9)\left(m^{2}+12 m\right.$ & $m^{4}+24 m^{3}+190 m^{2}+552 m+513$ & \\
\hline$(m+2)(m+10)\left(m^{2}+12 m\right.$ & $m^{4}+24 m^{3}+208 m^{2}+768 m+880$ & 2 \\
\hline$(m+6)^{2}\left(m^{2}+12 m+28\right)=$ & $m^{4}+24 m^{3}+208 m^{2}+768 m+1008$ & 6 \\
\hline$(m+4)^{2}(m+8)^{2}=$ & $m^{4}+24 m^{3}+208 m^{2}+768 m+1024$ & 4 \\
\hline & $m^{4}+24 m^{3}+208 m^{2}+768 m+1152$ & 12 \\
\hline
\end{tabular}

\begin{tabular}{|c|c|}
\hline $3 \quad 2 \quad 1$ & $\widetilde{n}=6$ \\
\hline$(m+1)(m+5)=m^{2}+6 m+5$ & 1 \\
\hline$(m+2)(m+4)=m^{2}+6 m+8$ & 2 \\
\hline$(m+3)^{2}=\quad m^{2}+6 m+9$ & 3 \\
\hline$m^{2}+6 m+12$ & 6 \\
\hline
\end{tabular}

\section{More observations}

The tables suggest more observations. For instance, the polynomials for which $\operatorname{gcd}\{m, \widetilde{n}\}=1$ have the smallest coefficients, those for which $\operatorname{gcd}\{m, \widetilde{n}\}=\widetilde{n}$ the biggest. This is of course what one expects.

There are $\left|\Delta^{(m-h)}\right|$ points in the "interior" of $\Delta^{(m)}$, i. e., in

$$
\left\{\lambda+\rho \mid \lambda \in P_{+},\left\langle\lambda+\rho, \theta_{\mathrm{s}}^{\vee}\right\rangle<m\right\},
$$

where $\rho=\sum_{i=1}^{l} \varpi_{i}$. In fact, the points in the "interior" correspond to strictly positive integral solutions to

$$
a_{1} n_{1}+\cdots+a_{l} n_{l}<m .
$$

There are $\left|\Delta^{(m-h)}\right|$ such solutions because $1+n_{1}+\cdots+n_{l}=h$. (The 1 accounts for the strict inequality in (5).) $\left|\Delta^{(m-h)}\right|$ is connected to the polynomial for $\left|\Delta^{(m)}\right|$ by reciprocity, i. e., Serre duality in the context of toric varieties. To be precise, let $p_{a}(x)$ be the polynomial for which $\left|\Delta^{(m)}\right|=p_{a}(m)$ if $m \equiv a(\bmod \widetilde{n})$ and $m \geqslant 0$. Then

$$
p_{a-h}(m-h)=(-1)^{l} p_{a}(-m) .
$$

In particular, we have $1=\operatorname{gcd}\{1, \widetilde{n}\}=\operatorname{gcd}\{1-h, \widetilde{n}\}$, whence

$$
p_{1}(m-h)=(-1)^{l} p_{1}(-m)
$$

or, using the observation (4),

$$
\prod_{i=1}^{l}\left(m-h+m_{i}\right)=\prod_{i=1}^{l}\left(m-m_{i}\right) .
$$

Hence Serre duality yields a fancy but amusing way of obtaining the symmetry $\left(h-m_{l}, \ldots, h-m_{1}\right)=\left(m_{1}, \ldots, m_{l}\right)$ of the set of exponents.

The reader may draw further observations from the tables, e.g., describe which linear factors occur. 


\section{The Other AFFine CoXeter-Dynkin Diagrams}

As already mentioned, the tables for $\widehat{B}_{l}, \widehat{\mathrm{C}}_{l}, \widehat{\mathrm{F}}_{4}$, and $\widehat{\mathrm{G}}_{2}$ give the number of dominant integral weights at level $m$ for the twisted affine Kac-Moody algebras. Physicists may be more interested in the untwisted case and want to count the number of primary fields for the WZNW conformal field theory models. The tables for these numbers are given below.

The left column in the table for $\widehat{X}_{l}^{\vee}$ contains the polynomials $\left(l ! \prod_{i=0}^{l} n_{i}\right)\left|\Delta^{(m)}\right|$ with $\left|\Delta^{(m)}\right|$ defined as in equation (2) for $n_{0}, \ldots, n_{l}$ as shown in the diagrams, and the number in the right column is $\operatorname{gcd}\{m, \widetilde{n}\}$. As before, $\widetilde{n}=\operatorname{lcm}\left\{n_{0}, \ldots, n_{l}\right\}$. Again, if the level $m$ satisfies $\operatorname{gcd}\{m, \widetilde{n}\}=1,\left|\Delta^{(m)}\right|$ is a product of $l$ linear factors. This time the connexion with the exponents is less straightforward.

The sum $g:=n_{0}+n_{1}+\cdots+n_{l}$ is the dual Coxeter number. Now the reciprocity formula connects $\left|\Delta^{(m-g)}\right|$ with the polynomial for $\left|\Delta^{(m)}\right|$.

Also there is the table for $\widehat{\mathrm{BC}}_{l}$ which merely counts lattice points for $\widehat{\mathrm{C}}_{l}$, but in a stuttering manner, meaning that $\left|\Delta^{(2 \mu+1)}\right|=\left|\Delta^{(2 \mu)}\right|$ for $\mu \in \mathbb{Z}$.

6. More tables

\begin{tabular}{|l|r|}
\hline$\widehat{\mathrm{B}}_{l}^{\vee}$ & \multicolumn{2}{|c|}{$\tilde{n}=2$} \\
\hline$\left(m+\frac{3}{2} l-1\right) \prod_{i=1}^{l-1}(m+2 i-1)$ & 1 \\
\hline$\left(m+\frac{l}{2}\right) \prod_{i=1}^{l-1}(m+2 i)$ & 2 \\
\hline
\end{tabular}

\begin{tabular}{|lrrrrr|r|}
\hline$\widehat{\mathrm{C}}_{l}^{\vee}$ & 1 & 1 & 1 & 1 & 1 & 1 \\
\hline same table as for $\widehat{\mathrm{A}}_{l}$ & & & & $\tilde{n}=1$ \\
\hline
\end{tabular}

\begin{tabular}{|c|c|c|}
\hline$\widehat{\mathrm{F}}_{4}^{\vee}$ & \begin{tabular}{lllll}
1 & 2 & 3 & 2 & 1 \\
\hdashline & 0 & 0
\end{tabular} & $\widetilde{n}=6$ \\
\hline$(m+1)(m+5)^{2}(m+7)=$ & $m^{4}+18 m^{3}+112 m^{2}+270 m+175$ & 1 \\
\hline$(m+3)\left(m^{3}+15 m^{2}+67 m\right.$ & $69)=m^{4}+18 m^{3}+112 m^{2}+270 m+207$ & 3 \\
\hline$(m+2)(m+4)^{2}(m+8)=$ & $m^{4}+18 m^{3}+112 m^{2}+288 m+256$ & 2 \\
\hline$(m+6)\left(m^{3}+12 m^{2}+40 m\right.$ & 48) $=m^{4}+18 m^{3}+112 m^{2}+288 m+288$ & 6 \\
\hline
\end{tabular}

\begin{tabular}{|llll|}
\hline$\widehat{\mathrm{G}}_{2}^{\vee}$ & 1 & 2 & 1 \\
\hline same table as for $\widehat{\mathrm{B}}_{2}$ & & $\tilde{n}=2$ \\
\hline
\end{tabular}

\begin{tabular}{|l|r|r|}
\hline$\widehat{\mathrm{BC}}_{l}$ & $222 \ldots 22$ & 2 \\
\hline$\prod_{i=1}^{l}(m+2 i-1)$ & 2 & 1 \\
\hline$\prod_{i=1}^{l}(m+2 i)$ & & 2 \\
\hline
\end{tabular}




\section{REFERENCES}

1. N. Bourbaki, Groupes et algèbres de Lie, Chapitres 4, 5 et 6 . Hermann, Paris 1968. Masson, Paris 1981. MR 39:1590

2. M. Brion, Points entiers dans les polytopes convexes, Séminaire Bourbaki (1993/94). Astérisque 227 (1995), exposé $\mathrm{n}^{\circ}$ 780, 145-169. MR 96e:11123

3. K. S. Brown, Buildings. Springer-Verlag, New York-Berlin 1989. MR 90e:20001

4. D. A. Cox, Recent developments in toric geometry. (to appear in the Proceedings of the 1995 Santa Cruz Summer Institute)

5. J. E. Humphreys, Reflection groups and Coxeter groups, Cambridge Studies in Advanced Mathematics 29. Cambridge University Press, Cambridge 1990. MR 92h:20002

Department of Mathematics, Massachusetts Institute of Technology, Cambridge, MASSACHUSETTS 02139-4307

Current address: Mathematik, Eidgenössische Technische Hochschule Zürich, ETH Zentrum, 8092 Zürich, Switzerland

E-mail address: suter@math.ethz.ch 